PROCEEDINGS OF THE

AMERICAN MATHEMATICAL SOCIETY

Volume 137, Number 11, November 2009, Pages 3627-3635

S 0002-9939(09)09950-X

Article electronically published on June 9, 2009

\title{
LYUBEZNIK RESOLUTIONS AND THE ARITHMETICAL RANK OF MONOMIAL IDEALS
}

\author{
KYOUKO KIMURA
}

(Communicated by Bernd Ulrich)

\begin{abstract}
In this paper, we prove that the length of a Lyubeznik resolution of a monomial ideal gives an upper bound for the arithmetical rank of the ideal.
\end{abstract}

\section{INTRODUCTION}

Let $S$ be a polynomial ring over a field $K$. Let $I$ be a monomial ideal of $S$ and $G(I)=\left\{m_{1}, m_{2}, \ldots, m_{\mu}\right\}$ the minimal set of monomial generators of $I$. In general, it is unknown how to construct a minimal graded free resolution of $S / I$. In 1960, Taylor 16 discovered a graded free resolution of $S / I$, which is called the Taylor resolution of $I$ :

$$
T_{\bullet}: 0 \longrightarrow T_{\mu} \stackrel{d_{\mu}}{\longrightarrow} T_{\mu-1} \stackrel{d_{\mu-1}}{\longrightarrow} \cdots \stackrel{d_{1}}{\longrightarrow} T_{0} \longrightarrow S / I \longrightarrow 0,
$$

where

$$
\begin{aligned}
T_{0}=S e_{\emptyset}, T_{s} & =\bigoplus_{1 \leq i_{1}<i_{2}<\cdots<i_{s} \leq \mu} S e_{i_{1} i_{2} \cdots i_{s}}, \\
d_{s}\left(e_{i_{1} i_{2} \cdots i_{s}}\right) & =\sum_{j=1}^{s}(-1)^{j-1} \frac{\operatorname{lcm}\left(m_{i_{1}}, \ldots, m_{i_{s}}\right)}{\operatorname{lcm}\left(m_{i_{1}}, \ldots, \widehat{m_{i_{j}}}, \ldots, m_{i_{s}}\right)} e_{i_{1} \cdots \widehat{i_{j}} \cdots i_{s}} .
\end{aligned}
$$

Here $e_{i_{1} i_{2} \cdots i_{s}}\left(1 \leq i_{1}<i_{2}<\cdots<i_{s} \leq \mu\right)$ are free basis elements of $T_{s}$, and the degree of $e_{i_{1} i_{2} \cdots i_{s}}$ is defined by

$$
\operatorname{deg} e_{i_{1} i_{2} \cdots i_{s}}=\operatorname{deg} \operatorname{lcm}\left(m_{i_{1}}, m_{i_{2}}, \ldots, m_{i_{s}}\right) .
$$

In 1988, Lyubeznik [13] constructed a graded free resolution of $S / I$ as a subcomplex of the Taylor resolution of $I$. This complex is called a Lyubeznik resolution.

We recall the definition of a Lyubeznik resolution. Let $1 \leq i_{1}<i_{2}<\cdots<i_{s} \leq$ $\mu$. If $m_{q}$ does not divide $\operatorname{lcm}\left(m_{i_{t}}, m_{i_{t+1}}, \ldots, m_{i_{s}}\right)$ for all $t<s$ and for all $q<i_{t}$, then the symbol $e_{i_{1} i_{2} \cdots i_{s}}$ is said to be L-admissible. The Lyubeznik resolution of $I$ is a subcomplex of the Taylor resolution of $I$ generated by all $L$-admissible symbols. Note that a Lyubeznik resolution of $I$ depends on the order of the generators $m_{1}, m_{2}, \ldots, m_{\mu}$. We define the L-length of $I$ as the minimum length of Lyubeznik resolutions of $I$. The Taylor resolution of $I$ is far from being a minimal graded free resolution in general, but a Lyubeznik resolution of $I$ often gives a minimal graded

Received by the editors December 1, 2008, and, in revised form, February 26, 2009.

2000 Mathematics Subject Classification. Primary 13E15; Secondary 13D02.

Key words and phrases. Lyubeznik resolution, $L$-admissible, $L$-length, arithmetical rank.

(C)2009 American Mathematical Society 3627

Reverts to public domain 28 years from publication 
free resolution or a graded free resolution whose length is equal to the projective dimension of $S / I$.

The arithmetical rank of $I$ is defined by

$\operatorname{ara} I:=\min \left\{r \in \mathbb{N}:\right.$ there exist $a_{1}, \ldots, a_{r} \in I$ such that $\left.\sqrt{\left(a_{1}, \ldots, a_{r}\right)}=\sqrt{I}\right\}$.

A trivial upper bound for ara $I$ is the cardinality of the minimal set of monomial generators of $I$, denoted by $\mu(I)=\mu$, which is equal to the length of the Taylor resolution of $I$. In this paper, we prove the following theorem:

Theorem 1. Let I be a monomial ideal of $S$. If the L-length of $I$ is $\lambda$, then

$$
\operatorname{ara} I \leq \lambda
$$

It is known from Lyubeznik [12] that if $I$ is a squarefree monomial ideal, then $\operatorname{pd}_{S} S / I \leq \operatorname{ara} I$, where $\operatorname{pd}_{S} S / I$ is the projective dimension of $S / I$. It is natural to ask when ara $I=\operatorname{pd}_{S} S / I$ holds for a squarefree monomial ideal $I$. The author together with Terai and Yoshida ([9, 10]; see also [11]) has proved that ara $I=$ $\operatorname{pd}_{S} S / I$ for squarefree monomial ideals $I$ with $\mu(I)-$ height $I \leq 2$. Barile [3, 4, 5, 6, 7], Barile and Terai [ 8 , and Schmitt and Vogel [15] also proved the same equality for some classes of squarefree monomial ideals. Since the projective dimension of $S / I$ is equal to the length of the minimal graded free resolution of $S / I$, we have the following corollary:

Corollary 2. Let I be a squarefree monomial ideal of $S$. If the L-length of I is equal to the projective dimension of $S / I$, then

$$
\operatorname{ara} I=\operatorname{pd}_{S} S / I \text {. }
$$

In particular, if the Lyubeznik resolution of I with respect to some order of monomial generators is minimal, then the same assertion is true.

In Section 2, we prove Theorem 1 and several corollaries. In Section 3, we give examples of squarefree monomial ideals $I$ whose $L$-length is equal to the projective dimension of $S / I$; see Barile [1, 2]. We also show that for the Stanley-Reisner ideal $I$ of the triangulation of the projective plane with 6 vertices, the $L$-length of $I$ coincides with ara $I$. Notice that Yan [17] proved that ara $I=4>3=\operatorname{pd}_{S} S / I$ when $\operatorname{char} K \neq 2$.

\section{Proof of Theorem 1}

In this section, we prove Theorem 1, which is the main result in this paper.

Proof of Theorem 1. Let $G(I)=\left\{m_{1}, m_{2}, \ldots, m_{\mu}\right\}$ be the minimal set of monomial generators of $I$. We consider the Lyubeznik resolution of $I$ with respect to this order.

To prove the theorem, it is enough to find $\lambda$ elements $g_{1}, g_{2}, \ldots, g_{\lambda}$ such that

$$
\sqrt{\left(g_{1}, g_{2}, \ldots, g_{\lambda}\right)}=\sqrt{I}
$$


We set

$$
\left\{\begin{array}{l}
g_{1}=m_{1}, \\
g_{2}=m_{2}+\sum_{\substack{\left[i_{1}, i_{2}, \ldots, i_{\lambda-1}\right] \in L_{\lambda-1} \\
i_{1} \geq 3}} m_{i_{1}} m_{i_{2}} \cdots m_{i_{\lambda-1}}, \\
\quad \vdots \\
g_{\ell}=m_{\ell}+\sum_{\substack{\left[i_{1}, i_{2}, \ldots, i_{\lambda-\ell+1}\right] \in L_{\lambda-\ell+1} \\
i_{1} \geq \ell+1}} m_{i_{1}} m_{i_{2}} \cdots m_{i_{\lambda-\ell+1},}, \\
g_{\lambda}=m_{\lambda}+\sum_{\substack{\left[i_{1}\right] \in L_{1} \\
i_{1} \geq \lambda+1}} m_{i_{1}}=m_{\lambda}+m_{\lambda+1}+\cdots+m_{\mu},
\end{array}\right.
$$

where

$$
L_{s}:=\left\{\left[i_{1}, i_{2}, \ldots, i_{s}\right] \in \mathbb{N}^{s}: \begin{array}{l}
1 \leq i_{1}<i_{2}<\cdots<i_{s} \leq \mu \\
e_{i_{1} i_{2} \cdots i_{s}} \text { is } L \text {-admissible }
\end{array}\right\}
$$

Put $J=\left(g_{1}, g_{2}, \ldots, g_{\lambda}\right)$. We prove that $m_{\ell} \in \sqrt{J}$ for all $\ell=1,2, \ldots, \mu$ by induction on $\ell$. We need the following lemma:

Lemma 3. Suppose $\left[i_{1}, i_{2}, \ldots, i_{s}\right] \in L_{s}$. Then:

(1) $\left[i_{j_{1}}, \ldots, i_{j_{t}}\right] \in L_{t}$ for all $t \leq s$ and for all $1 \leq j_{1}<\cdots<j_{t} \leq s$.

(2) If $i_{1}>1$, then $\left[1, i_{1}, i_{2}, \ldots, i_{s}\right] \in L_{s+1}$. In particular, if $\left[i_{1}, i_{2}, \ldots, i_{\lambda}\right] \in L_{\lambda}$, then $i_{1}=1$.

(3) Suppose $\ell<i_{1}$. If $\left[\ell, i_{1}, i_{2}, \ldots, i_{s}\right] \notin L_{s+1}$, then $m_{\ell} m_{i_{1}} m_{i_{2}} \cdots m_{i_{s}}$ is divisible by at least one of $m_{1}, m_{2}, \ldots, m_{\ell-1}$.

Proof. These follow from the definition of $L$-admissibleness.

The case $\ell=1$ is clear because $m_{1}=g_{1}$. For $\ell=2$, we consider $m_{2} g_{2}$. Then

$$
m_{2} g_{2}=m_{2}^{2}+\sum_{\substack{\left[i_{1}, i_{2}, \ldots, i_{\lambda-1}\right] \in L_{\lambda-1} \\ i_{1} \geq 3}} m_{2} m_{i_{1}} m_{i_{2}} \cdots m_{i_{\lambda-1}} \in J .
$$

Since $\left[2, i_{1}, i_{2}, \ldots, i_{\lambda-1}\right] \notin L_{\lambda}$ by Lemma 3 (2), the second term is divisible by $m_{1}$ by Lemma 3 (3). Hence $m_{2}^{2} \in J$, and thus $m_{2} \in \sqrt{J}$.

We assume $\ell>2$ and $m_{1}, m_{2}, \ldots, m_{\ell-1} \in \sqrt{J}$. Set $\nu=\nu_{\ell}=\min \{\ell-2, \lambda-2\}$. Then we show that

$$
\sum_{\left[\ell, i_{2}, \ldots, i_{s}\right] \in L_{s}} m_{\ell} m_{i_{2}} \cdots m_{i_{s}} \in \sqrt{J}
$$

by descending induction on $s(\lambda-\nu \leq s \leq \lambda-1)$.

First, we consider $m_{\ell} g_{2}$. By a similar argument as in the case $\ell=2$, we have (2.1) for $s=\lambda-1$.

Next, we assume

$$
\sum_{\left[\ell, i_{2}, \ldots, i_{s+1}\right] \in L_{s+1}} m_{\ell} m_{i_{2}} \cdots m_{i_{s+1}} \in \sqrt{J}
$$


and prove (2.1). Then $m_{\ell} g_{\lambda-s+1} \in J$ implies that

$$
m_{\ell} m_{\lambda-s+1}+\sum_{\substack{\left[i_{1}, i_{2}, \ldots, i_{s}\right] \in L_{s} \\ i_{1} \geq \lambda-s+2}} m_{\ell} m_{i_{1}} m_{i_{2}} \cdots m_{i_{s}} \in J .
$$

Since $\lambda-s+1 \leq \nu+1<\ell$ by the definition of $\nu$, we have

$$
\sum_{\left[\ell, i_{2}, \ldots, i_{s}\right] \in L_{s}} m_{\ell}^{2} m_{i_{2}} \cdots m_{i_{s}}+\sum_{\substack{\left[i_{1}, i_{2}, \ldots, i_{s}\right] \in L_{s} \\ i_{1}>\ell}} m_{\ell} m_{i_{1}} m_{i_{2}} \cdots m_{i_{s}} \in \sqrt{J} .
$$

The second term can be written in the following form:

$$
\sum_{\left[\ell, i_{1}, i_{2}, \ldots, i_{s}\right] \in L_{s+1}} m_{\ell} m_{i_{1}} m_{i_{2}} \cdots m_{i_{s}}+\sum_{\substack{\left[i_{1}, i_{2}, \ldots, i_{s}\right] \in L_{s} \\\left[\ell, i_{1}, i_{2}, \ldots, i_{s}\right] \notin L_{s+1}}} m_{\ell} m_{i_{1}} m_{i_{2}} \cdots m_{i_{s}} .
$$

The first term of (2.3) is in $\sqrt{J}$ by assumption (2.2). The second term of (2.3) is in $\sqrt{J}$ by Lemma 3 (3). Therefore (2.1) is also satisfied for $s$. Hence, (2.1) is satisfied for all $s \geq \lambda-\nu$.

Now, we prove that $m_{\ell} \in \sqrt{J}$. If $\nu=\ell-2$, then we consider $m_{\ell} g_{\ell}$. By a similar argument as above, we have

$$
m_{\ell}^{2}+\sum_{\left[\ell, i_{1}, i_{2}, \ldots, i_{\lambda-\ell+1}\right] \in L_{\lambda-\ell+2}} m_{\ell} m_{i_{1}} m_{i_{2}} \cdots m_{i_{\lambda-\ell+1}} \in \sqrt{J} .
$$

Since (2.1) is satisfied for $s=\lambda-\nu=\lambda-\ell+2$, we have $m_{\ell}^{2} \in \sqrt{J}$ and so $m_{\ell} \in \sqrt{J}$ as required. For $\nu=\lambda-2$, we consider $m_{\ell} g_{\lambda}$. By a similar argument as in the case of $\nu=\ell-2$, we have $m_{\ell} \in \sqrt{J}$.

Proof of Corollary 2, By Lyubeznik [12, we have $\operatorname{pd}_{S} S / I \leq$ ara $I$. On the other hand, our theorem gives the opposite inequality.

We also have an upper bound on the arithmetical rank, which was proved by Terai.

Corollary 4 (Terai). Let $I$ be a squarefree monomial ideal of $S$, and let $G(I)=$ $\left\{m_{1}, m_{2}, \ldots, m_{\mu}\right\}$ be the minimal set of monomial generators of $I$. We set

$$
l=\max \left\{l: \begin{array}{c}
m_{j_{1}} \neq \operatorname{lcm}\left(m_{j_{1}}, m_{j_{2}}\right) \neq \cdots \neq \operatorname{lcm}\left(m_{j_{1}}, m_{j_{2}}, \ldots, m_{j_{s}}\right) \\
\text { for some } m_{j_{1}}, m_{j_{2}}, \ldots, m_{j_{s}} \in G(I)
\end{array}\right\} .
$$

Then we have

$$
\operatorname{ara} I \leq l \text {. }
$$

Proof. Let $\lambda$ denote the length of a Lyubeznik resolution of $I$. If $e_{i_{1} i_{2} \cdots i_{\lambda}}$ is $L$ admissible, then

$$
m_{i_{\lambda}} \neq \operatorname{lcm}\left(m_{i_{\lambda}}, m_{i_{\lambda-1}}\right) \neq \cdots \neq \operatorname{lcm}\left(m_{i_{\lambda}}, m_{i_{\lambda-1}}, \ldots, m_{i_{1}}\right)
$$

by the definition. Therefore $\lambda \leq l$ holds and Corollary 2 gives the desired inequality.

The next corollary was proved by Barile [1, 2]. 
Corollary 5 (Barile [1, Proposition 2.4], 2, Remark 3]). Let I be a squarefree monomial ideal and let $G(I)=\left\{m_{1}, m_{2}, \ldots, m_{\mu}\right\}$ be the minimal set of monomial generators of $I$. If there exists an integer $s>1$ such that $m_{1}$ divides $m_{i_{1}} \cdots m_{i_{s}}$ for all $2 \leq i_{1}<\cdots<i_{s} \leq \mu$, then

$$
\text { ara } I \leq s
$$

Proof. The assumption implies that $L_{s+1}=\emptyset$. Then the assertion follows from Corollary 2

\section{EXAmples}

In this section, we give some examples of Lyubeznik resolutions.

For two $L$-admissible symbols $e_{i_{1} \cdots i_{s}}$ and $e_{j_{1} \cdots j_{t}}$, we say that

$$
e_{i_{1} \cdots i_{s}} \leq e_{j_{1} \cdots j_{t}}
$$

if $i_{1}, \ldots, i_{s}$ is a subsequence of $j_{1}, \ldots, j_{t}$. This induces a partial order on the set of all $L$-admissible symbols. Barile [2, Remark 1] pointed out that a necessary and sufficient condition for a Lyubeznik resolution of $I$ to be minimal is that for all maximal $L$-admissible symbols $e_{i_{1} \cdots i_{s}}$,

$$
\operatorname{lcm}\left(m_{i_{1}}, \ldots, m_{i_{s}}\right) \neq \operatorname{lcm}\left(m_{i_{1}}, \ldots, \widehat{m_{i_{j}}}, \ldots, m_{i_{s}}\right) \quad \text { for all } j=1, \ldots, s .
$$

First, we consider an ideal $I$ whose Lyubeznik resolution is minimal. The first example shows that a Lyubeznik resolution of $I$ is minimal for an ideal $I$ with $\mu(I)-$ height $I \leq 1$.

Example 6 (See 9, Theorem 2.1]). Let $I$ be a squarefree monomial ideal with $\mu(I)-\operatorname{pd}_{S} S / I \leq 1$. Then the $L$-length of $I$ is equal to $\operatorname{pd}_{S} S / I$. In particular, we have ara $I=\operatorname{pd}_{S} S / I$ by Corollary 2 ,

Moreover we assume that $\mu(I)-$ height $I \leq 1$. The author classified these ideals in [9, Theorem 4.4] with Terai and Yoshida. Then it is easy to see that a Lyubeznik resolution of $I$ is minimal.

Remark 7. For the ideal $I$ in Example 6, there are many proofs of ara $I=\operatorname{pd}_{S} S / I$. For example, we can also prove it by the method of Barile [2, Proposition 2].

When $\mu(I)$ - height $I=2$, a Lyubeznik resolution of $I$ is not necessarily minimal as the next example shows.

Example 8. Let $I=\left(m_{1}, m_{2}, m_{3}, m_{4}\right)$ be a squarefree monomial ideal with $\mu(I)$ height $I=2$. Assume that $S / I$ is Cohen-Macaulay.

If $m_{1}$ divides $m_{i} m_{j}$ for all $2 \leq i<j \leq 4$ upon renumbering the generators, then the Lyubeznik resolution of $I$ with respect to this order is minimal. Otherwise, the $L$-length of $I$ is larger than the projective dimension of $S / I$, and thus Lyubeznik resolutions of $I$ are not minimal for any order of generators.

Note that in both cases, $\operatorname{ara} I=\operatorname{pd}_{S} S / I=2$ holds by [10, Proposition 4.5].

The next example was considered by Barile [1]. 
Example 9 (Barile [1, Example 2.6]). Let $I$ be the squarefree monomial ideal generated by the following $n+2$ elements:

$$
\left\{\begin{aligned}
m_{i} & =x_{1} x_{2} x_{2 i+1} x_{2 i+2}, \quad i=1,2, \ldots, n-1, \\
m_{n} & =x_{1} x_{3} x_{5} \cdots x_{2 n-1} x_{2 n+1} \\
m_{n+1} & =x_{1} x_{4} x_{6} x_{8} \cdots x_{2 n-2} x_{2 n} x_{2 n+1} \\
m_{n+2} & =x_{2} x_{3} \cdots x_{2 n} x_{2 n+1} .
\end{aligned}\right.
$$

Barile [1, 2] proved that $\operatorname{ara} I=\operatorname{pd}_{S} S / I=n$. She computed $\operatorname{pd}_{S} S / I$ by proving that the Lyubeznik resolution of $I$ with this order is minimal.

For another example, Novik [14 proved that a Lyubeznik resolution is minimal for the matroid ideal of a finite projective space.

Secondly, we exhibit several ideals whose Lyubeznik resolutions are not necessarily minimal, but which have $L$-length equal to the projective dimension. Let $\lambda$ be the length of the Lyubeznik resolution of $I$ with respect to some order of monomial generators of $I$. A sufficient condition for $\lambda=\operatorname{pd}_{S} S / I$ to hold is that one of the $L$-admissible symbols $e_{i_{1} \cdots i_{\lambda}}$ must satisfy

$$
\operatorname{lcm}\left(m_{i_{1}}, \ldots, m_{i_{\lambda}}\right) \neq \operatorname{lcm}\left(m_{i_{1}}, \ldots, \widehat{m_{i_{j}}}, \ldots, m_{i_{\lambda}}\right) \quad \text { for all } j=1, \ldots, \lambda .
$$

The next example is a generalization of [10, Lemma 5.1].

Example 10. Let $I=\left(m_{1}, m_{2}, \ldots, m_{\mu}\right)$ be a squarefree monomial ideal with $\mu(I)-\operatorname{pd}_{S} S / I=2$. We assume that $m_{i} m_{j}$ is divisible by one of $m_{1}, m_{2}, \ldots, m_{\mu-3}$ for all $\mu-2 \leq i<j \leq \mu$. Then the $L$-length of $I$ is equal to $\operatorname{pd}_{S} S / I$. In particular, we have ara $I=\operatorname{pd}_{S} S / I$ by Corollary 2

Remark 11. For an ideal $I$ as in Example 10, we also have ara $I=\operatorname{pd}_{S} S / I$ by the result of Schmitt-Vogel [15, Lemma].

The next example was considered by Barile [1, Example 2.7].

Example 12 (Barile 1, Example 2.7]). Let $I$ be the squarefree monomial ideal generated by the following 8 elements:

$$
x_{1} x_{2} x_{3}, x_{1} x_{4} x_{5} x_{6}, x_{2} x_{7}, x_{3} x_{8}, x_{1} x_{9}, x_{4} x_{10}, x_{5} x_{11}, x_{6} x_{12} .
$$

Barile proved that ara $I=\operatorname{pd}_{S} S / I=6$.

In the left (resp. right) table below, the element in the $i$ th column and $j$ th row is $\beta_{i, i+j}^{S}(S / I):=\operatorname{dim}_{K}\left[\operatorname{Tor}_{S}^{i}(K, S / I)\right]_{i+j}$ (resp. the cardinality of the set $\left[t_{1}, \cdots, t_{i}\right] \in$ $\left.\left.L_{i}: \operatorname{deg} e_{t_{1} \cdots t_{i}}=i+j\right\}\right)$ :

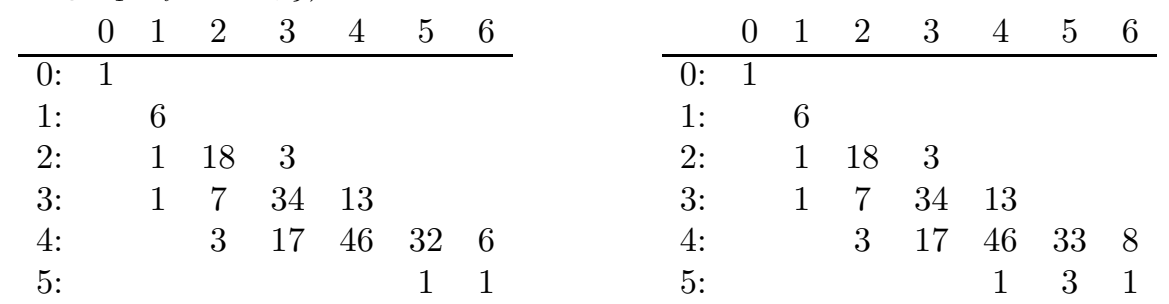

The difference between these tables arises from the $L$-admissibility of $e_{124678}$, $e_{123678}$ and $e_{12678}$. As these tables show, the Lyubeznik resolution of $I$ is not minimal, but the $L$-length of $I$ is equal to $\operatorname{pd}_{S} S / I=6$.

The next example is a generalization of the ideals in [10, Subsection 4.4]. 
Example 13. Let $j, k, \ell, n$ be integers with $1 \leq j \leq k \leq \ell<n-2$. Let $I$ be the squarefree monomial ideal generated by the following $n$ elements:

$$
\begin{aligned}
m_{1} & =x_{1} \cdots x_{k} y_{\ell+1} \cdots y_{n-2}, \\
m_{2} & =x_{1} \cdots x_{k} y_{j} \cdots y_{\ell}, \\
m_{i+2} & =x_{i} y_{i} z_{t_{i}}, \quad 1 \leq i \leq n-2 .
\end{aligned}
$$

Set $x_{i}=1$ for $k<i \leq n-2$ and $y_{i}=1$ for $1 \leq i<j$. Then the product $m_{3} \cdots m_{n}$ is divisible by $m_{1}$. We consider the product $m_{3} \cdots \widehat{m_{i}} \cdots m_{n}$. When $i \leq \ell$, a product $m_{2} m_{3} \cdots \widehat{m_{i}} \cdots m_{n}$ is divisible by $m_{1}$. When $i>\ell$, a product $m_{3} \cdots \widehat{m_{i}} \cdots m_{n}$ is divisible by $m_{2}$. This means that the $L$-length of $I$ is at most $n-2$. Hence ara $I \leq n-2$.

In particular, we have ara $I=\operatorname{pd}_{S} S / I=n-2$ for the following cases:

(1) $z_{t_{i}}=z_{i}$ for all $i=1,2, \ldots, n-2$.

(2) $z_{t_{k}}=z_{t_{n-2}}=z_{k}$, and $z_{t_{i}}=z_{i}$ for $i \neq k, n-2$.

In fact, the ideal in case (1) satisfies $\mu(I)$ - height $I=2$ (see [10, Subsection 4.4]). In case (2), $e_{1 \cdots \widehat{k+2} \cdots n-1}$ is $L$-admissible and satisfies (3.1).

Remark 14. Let $m_{1}, m_{2}, \ldots, m_{n}$ be squarefree monomials as in Example 13 and let $w$ be a new variable. Put $T \subset\{3,4, \ldots, n\}$ with $\sharp T \geq 2$. We set $I^{\prime}=$ $\left(m_{1}^{\prime}, m_{2}^{\prime}, \ldots, m_{n}^{\prime}\right)$, where

$$
\begin{cases}m_{1}^{\prime}=m_{1} w, & \\ m_{2}^{\prime}=m_{2}, & \\ m_{i}^{\prime}=m_{i} w, & \text { if } i \in T, \\ m_{i}^{\prime}=m_{i}, & \text { if } i \in\{3,4, \ldots, n\} \backslash T .\end{cases}
$$

Then the same assertion as in Example 13 is true. For example, for the ideal $I^{\prime}$ generated by the following 6 elements, we have ara $I^{\prime}=\operatorname{pd}_{S} S / I^{\prime}=4$ :

$$
x_{1} x_{2} y_{4} w, x_{1} x_{2} y_{2} y_{3}, x_{1} z_{1} w, x_{2} y_{2} z_{2} w, y_{3} z_{3}, y_{4} z_{2} \text {. }
$$

Moreover, for this ideal $I^{\prime}$, it seems to be difficult to show ara $I^{\prime}=\operatorname{pd}_{S} S / I^{\prime}$ by the method of Barile ([1, Proposition 1.1], [2, Propositions 1, 2]).

Finally, we give an ideal $I$ whose $L$-length is not equal to the projective dimension of $S / I$ but is equal to the arithmetical rank of $I$. In the following example, we consider the Stanley-Reisner ideal $I$ of the triangulation of the projective plane with 6 vertices. The projective dimension of $S / I$ depends on the characteristic of $K$, and Yan [17] proved that ara $I=4>3=\operatorname{pd}_{S} S / I$ if char $K \neq 2$. Our theorem provides the best upper bound for ara $I$.

Example 15 (Yan [17]). Let $I$ be the squarefree monomial ideal generated by the following 10 elements:

$$
x_{1} x_{2} x_{3}, x_{1} x_{2} x_{5}, x_{1} x_{3} x_{6}, x_{1} x_{4} x_{5}, x_{1} x_{4} x_{6}, x_{2} x_{3} x_{4}, x_{2} x_{4} x_{6}, x_{2} x_{5} x_{6}, x_{3} x_{4} x_{5}, x_{3} x_{5} x_{6} .
$$

This ideal is the Stanley-Reisner ideal of the triangulation of the projective plane with 6 vertices. Then a minimal graded free resolution of $I$ is given by the following left (resp. right) diagram if char $K \neq 2$ (resp. if char $K=2$ ): 


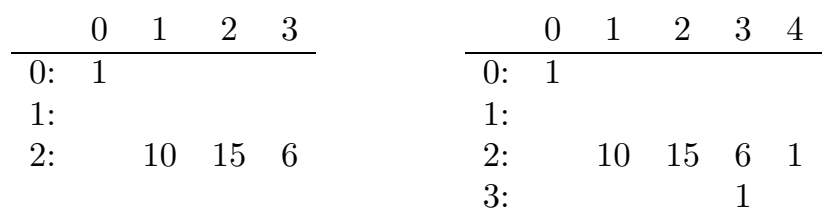

Hence, the projective dimension of $S / I$ is given by

$$
\operatorname{pd}_{S} S / I= \begin{cases}3 & \text { if } \operatorname{char} K \neq 2 \\ 4 & \text { if } \operatorname{char} K=2\end{cases}
$$

Yan 17. proved that ara $I=4$ for any characteristic of $K$.

On the other hand, the Lyubeznik resolution of $I$ with respect to this order is given by the following diagram:

\begin{tabular}{cccccc} 
& 0 & 1 & 2 & 3 & 4 \\
\hline $0:$ & 1 & & & & \\
1: & & & & & \\
2: & & 10 & 15 & 18 & 9 \\
3: & & & 12 & 9 &
\end{tabular}

In particular, the length is 4 . Therefore Theorem 1 implies that the $L$-length of $I$ coincides with ara $I$.

\section{ACKNOWLEDGMENTS}

The author is grateful to Professors Ken-ichi Yoshida and Naoki Terai for useful discussions.

\section{REFERENCES}

[1] M. Barile, On the number of equations defining certain varieties, Manuscripta Math. 91 (1996), 483-494. MR:1421287 (97m:13041)

[2] M. Barile, On ideals whose radical is a monomial ideal, Comm. Algebra 33 (2005), 44794490. MR2188323 (2006g:13039)

[3] M. Barile, A note on monomial ideals, Arch. Math. (Basel) 87 (2006), 516-521. MR2283682 (2007h:13004)

[4] M. Barile, A note on the edge ideals of Ferrers graphs, preprint, arXiv:math.AC/0606353.

[5] M. Barile, On the arithmetical rank of the edge ideals of forests, Comm. Algebra 36 (2008), 4678-4703. MR2473354

[6] M. Barile, On the arithmetical rank of certain monomial ideals, preprint, arXiv:math.AC/0611790.

[7] M. Barile, Arithmetical ranks of Stanley-Reisner ideals via linear algebra, Comm. Algebra 36 (2008), 4540-4556. MR.2473347

[8] M. Barile and N. Terai, Arithmetical ranks of Stanley-Reisner ideals of simplicial complexes with a cone, preprint, arXiv:0809.2194.

[9] K. Kimura, N. Terai, and K. Yoshida, Arithmetical rank of squarefree monomial ideals of small arithmetic degree, J. Algebraic Combin. 29 (2009), 389-404.

[10] K. Kimura, N. Terai, and K. Yoshida, Arithmetical rank of squarefree monomial ideals of deviation two, submitted.

[11] K. Kimura, N. Terai, and K. Yoshida, Arithmetical rank of squarefree monomial ideals whose Alexander duals have deviation two, in preparation.

[12] G. Lyubeznik, On the local cohomology modules $H_{\mathfrak{a}}^{i}(R)$ for ideals a generated by monomials in an R-sequence, in Complete Intersections, Acireale, 1983 (S. Greco and R. Strano, eds.), Lecture Notes in Mathematics, No. 1092, Springer-Verlag, 1984, pp. 214-220. MR775884 (86f:14002)

[13] G. Lyubeznik, A new explicit finite free resolution of ideals generated by monomials in an R-sequence, J. Pure Appl. Algebra 51 (1988), 193-195. MR941900 (89c:13020) 
[14] I. Novik, Lyubeznik's resolution and rooted complexes, J. Algebraic Combin. 16 (2002), 97101. MR.1941987 (2003j:13021)

[15] T. Schmitt and W. Vogel, Note on set-theoretic intersections of subvarieties of projective space, Math. Ann. 245 (1979), 247-253. MR553343 (81a:14025)

[16] D. Taylor, Ideals generated by monomials in an R-sequence, Ph.D. Thesis, Chicago University (1960).

[17] Z. Yan, An étale analog of the Goresky-MacPherson formula for subspace arrangements, J. Pure Appl. Algebra 146 (2000), 305-318. MR.1742346 (2000k:14041)

Graduate School of Mathematics, Nagoya University, Nagoya 464-8602, Japan

E-mail address: m04012w@math.nagoya-u.ac.jp

Current address: Department of Pure and Applied Mathematics, Graduate School of Information Science and Technology, Osaka University, Toyonaka, Osaka 560-0043, Japan

E-mail address: kimura@math.sci.osaka-u.ac.jp 